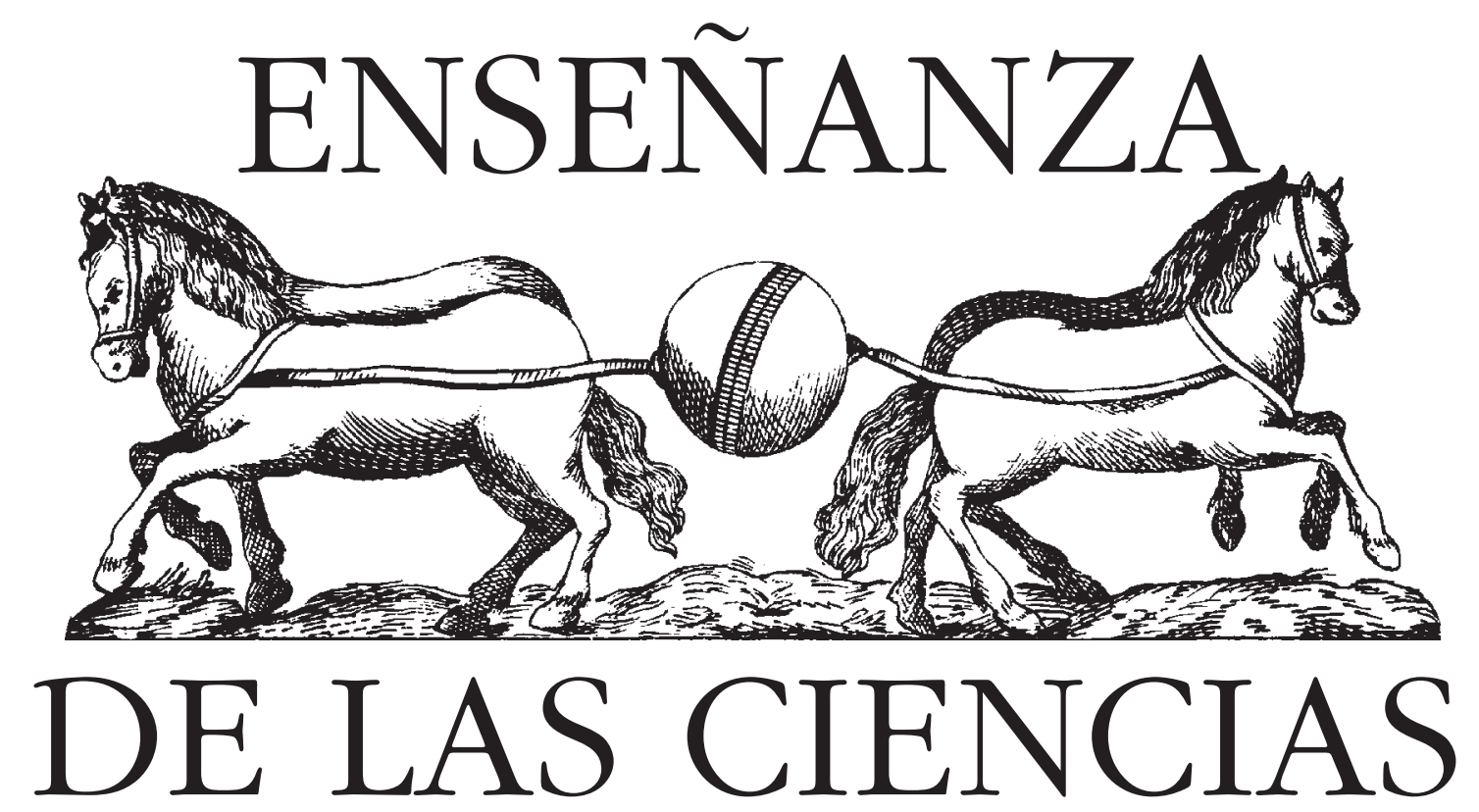

Revista de investigación y experiencias didácticas 


\section{NORMAS PARA LA PRESENTACIÓN DE ORIGINALES}

1) Los artículos se remitirán por correo electrónico $<$ r.ensenanza.ciencias@uab.es> En caso excepcional se pueden enviar por correo postal, una copia y disquete, a la siguiente dirección: Enseñanza de las Ciencias. Institut de Ciències de l'Educació. Edificio A. Universitat Autònoma de Barcelona. 08193 Bellaterra (Barcelona). En el artículo deberá figurar el nombre completo de los autores, la dirección, el correo electrónico y su lugar de trabajo.

2) Los artículos tendrán una extensión máxima de 45.000 caracteres, incluidas las tablas, las figuras y los anexos.

3) Junto con el artículo se remitirá un resumen (máximo de 10 líneas), una traducción del mismo en inglés, cinco palabras clave (en castellano e inglés) y el título del artículo también en inglés.

4) Se recomienda la confección de los originales con un procesador de textos (compatible para Macintosh y PC), de manera que, una vez aceptado el artículo, los autores lo remitan a la revista en la versión definitiva. Es imprescindible indicar el programa de tratamiento de textos utilizado.

5) Es imprescindible que los esquemas, dibujos, gráficas e imágenes sean guardados en formato TIF, EPS o JPEG. Éstos se adjuntarán en una carpeta aparte del documento del texto, ya que las imágenes incrustadas en el texto no son válidas para su posterior edición.

6) Todas las citas bibliográficas se relacionarán al final del artículo por orden alfabético de apellidos, indicando: autor(es), año, título de la revista completo y en cursiva (o subrayado), volumen, número y páginas del mismo. Por ejemplo:

NOVAK, J.D. (1977). An Alternative to Piagetian Psychology for Science and Mathematics Education. Science Education, 61(4), pp. 453-457.

En el caso de referencias de libros, se escribirá el título en cursiva (o subrayado) y se pondrá: el lugar de edición, dos puntos y la editorial. Por ejemplo:

OSBORNE, R. y FREYBERG, P. (1991). El aprendizaje de las ciencias. Implicaciones en la ciencia de los alumnos. Madrid: Narcea ediciones.

Todas las referencias bibliográficas deben corresponder a menciones hechas en el texto.

7) Dentro del texto, las referencias se indicarán dando, entre paréntesis, los apellido(s) de los autores o autoras y el año de publicación, separados por una coma. Por ejemplo: [...] obstáculo epistemológico (Bachelard, 1938).

Cuando el nombre del autor aparezca explícitamente en el texto, se pondrá el año del trabajo entre paréntesis. Por ejemplo: [...] según Bachelard (1938).

8) Los resúmenes de tesis didácticas se remitirán también por correo electrónico <r.ensenanza.ciencias@uab.es> Constarán de los siguientes datos: título, autor o autora, tipo de tesis (doctoral o de maestría), director(es) o directora(s), departamento, universidad, programa en el cual se ha presentado, fecha de presentación. La extensión máxima será de 4.500 caracteres. 


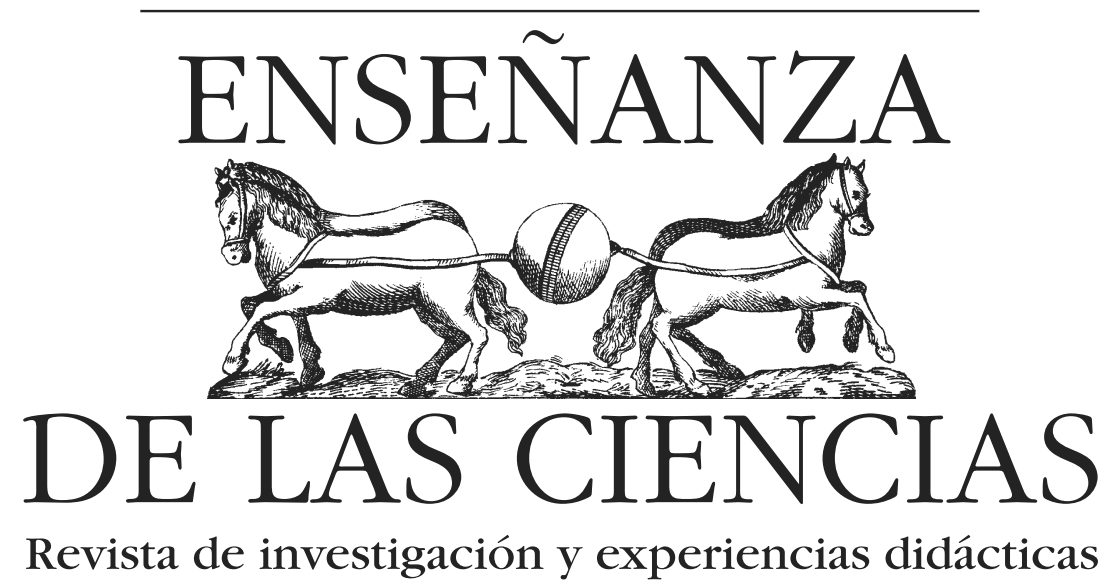

\section{CONSEJO DE REDACCIÓN}

Directoras: Carmen AZCÁRATE y Mercè IZQUIERDO. Departament de Didàctica de la Matemàtica $\mathrm{i}$ de les Ciències Experimentals de la Universitat Autònoma de Barcelona.

Óscar BARBERÁ. Departament de Didàctica de les Ciències Experimentals de la Universitat de València.

Mariona ESPINET. Departament de Didàctica de la Matemàtica i de les Ciències Experimentals de la Universitat Autònoma de Barcelona.

Carles FURIÓ. Departament de Didàctica de les Ciències Experimentals de la Universitat de València.

Conxita MÁRQUEZ. Departament de Didàctica de la Matemàtica i de les Ciències Experimentals de la Universitat Autònoma de Barcelona.

Luis PUIG. Departament de Didàctica de la Matemàtica de la Universitat de València.

Neus SANMARTÍ. Directora del ICE de la Universitat Autònoma de Barcelona.

Dora CARRERA. ICE de la Universitat Autònoma de Barcelona.

Colaboradores: Modesto SIERRA. Departamento de Didáctica de la Matemática y de las Ciencias Experimentales de la Universidad de Salamanca. Roque JIMÉNEZ PÉREZ. Departamento de Didáctica de las Ciencias de la Universidad de Huelva. Presidente de APICE (Asociación Española de Profesores e Investigadores en Didáctica de las Ciencias Experimentales).

\section{CONSEJO ASESOR}

Jean Pierre ASTOLFI, Institut National de Recherche Pédagogique. París (Francia)

Joan BACH, Dep. de Geologia. Universitat Autònoma de Barcelona. Enrique BANET, Dep. de Didáctica de las Ciencias. Universidad de Murcia. Guy BROUSSEAU, Profesor Emérito. Didactique et Anthropologie des Enseignements Scientifiques et Techniques (DAEST). Université Victor Segalen.

António CACHAPUZ, Dep. de Didáctica e Tecnologia Educativa. Universidade de Aveiro (Portugal).

Juan Manuel CAMPANARIO, Dep. de Física. Universidad de Alcalá de Henares.

Pedro CAÑAL, Dep. de Didáctica de las Ciencias. Universidad de Sevilla. Jaime CARRASCOSA, IES El Cid. València.

Fernando CERDÁN, Dep. de Didàctica de la Matemàtica. Universitat de València.

Leonor COLOMBO, Instituto de Física. Universidad Nacional de Tucumán (Argentina).

Moisés CORIAT, Dep. de Didáctica de la Matemáticas. Universidad de Granada.

Eugenio FILLOY, Departamento de Matemática Educativa. Centro de Investigación y Estudios Avanzados del Instituto Politécnico Nacional de México.

Daniel GIL, Dep. de Didàctica de les Ciències Experimentals. Universitat de València.

André GIORDAN, Laboratoire de Didactique et Epistémologie des Sciences. Ginebra (Suiza).

Ricard GUERRERO, Dep. de Microbiologia. Universitat de Barcelona. Jenaro GUISASOLA, Dep. de Física Aplicada I. Euskal Herriko Unibertsitatea-Universidad del País Vasco.

Ma Pilar JIMÉNEZ, Dep. de Didáctica de las Ciencias Experimentales. Universidad de Santiago de Compostela.

José LILLO, Dep. de Didáctica de las Ciencias Experimentales. Universidad de Vigo.

Salvador LLINARES, Dep. de Innovación y Formación Didáctica. Universitat d'Alacant.
Joan Antoni LLORENS, Dep. de Química. Universitat Politècnica de València.

Jean Louis MARTINAND, Laboratoire de Recherche en Education Scientifique et Technologique (LIRES). Ecole Normale Supérieure de Cachan (Francia).

Mercedes MARTíNEZ, Dep. de Didáctica de las Ciencias Experimentales. Universidad Complutense de Madrid.

Joaquín MARTÍNEZ TORREGROSA, Universitat d'Alacant.

Isabel MARTINS, Núcleo de Tecnologia Educacional para a Saúde. Universidade Federal do Rio de Janeiro (Brasil).

Vicente MELLADO, Dep. de Didáctica de las Ciencias Experimentales y de las Matemáticas. Universidad de Extremadura.

Marco A. MOREIRA, Instituto de Física. Universidade Federal do Rio Grande do Sud (Brasil).

Emilio PEDRINACI, IES de Gines. Sevilla.

Francisco J. PERALES, Dep. de Didáctica de las Ciencias Experimentales. Universidad de Granada.

Rafael PORLÁN, Dep. de Didáctica de las Ciencias. Universidad de Sevilla.

Antonio de PRO, Dep. de Didáctica de las Ciencias. Universidad de Murcia.

Luis RICO, Dep. de Didáctica de la Matemática. Universidad de Granada.

Julia SALINAS, Dep. de Física de la Facultad de Ciencias Exactas y Tecnología. Universidad Nacional de Tucumán (Argentina).

Alan H. SCHOENFELD, Graduate School of Education. University of California, Berkeley (California).

José Maria SEBASTIA, Dep. de Física. Universidad Simón Bolívar, Caracas (Venezuela).

Jordi SOLBES, IES José Rodrigo Botet. Manises.València.

Francisco TOMAS, Dep. de Química-Física. Universitat de València. Laurence VIENNOT, Laboratoire de Didactique de la Phisique dans l'Enseignement Supérieur. Université Paris VII (Francia).

Rod WATSON, Department of Education. King's College University of London (Londres). 


\section{NUEVOS SUSCRIPTORES}

Quienes deseen iniciar su suscripción a ENSEÑANZA DE LAS CIENCIAS pueden fotocopiar y rellenar este boletín y remitirlo a la siguiente dirección: ENSEÑANZA DE LAS CIENCIAS. Universitat Autònoma de Barcelona. Institut de Ciències de l'Educació. Edificio A. 08193 Bellaterra (Barcelona)

\section{BOLETÍN DE SUSCRIPCIÓN}

Formato papel: Año 2006

INDIVIDUAL

España

Otros países

35 Euros*

58 Euros

\section{Formato electrónico: Año 2006}

España y otros países 27 Euros

* IVA incluido

Señalar el o los formatos que suscribe: $\square$ Formato papel Formato electrónico

CIF:

E-mail:

\begin{tabular}{ll} 
Apellidos: & Nombre: \\
\hline Calle o plaza: & Núm.: \\
\hline Población: & D.P.: \\
\hline Provincia: & \\
\hline Profesión: & Tel.: \\
\hline
\end{tabular}

Especialidad:

Nivel educativo:

Firma

Fecha:

\section{PARA SUSCRIPTORES FUERA DE ESPAÑA}

VISA PREMIER/CLASSIC. EUROCARD/MASTERCARD

Autorizo a la Revista Enseñanza de las Ciencias de la Universitat Autònoma de Barcelona a cargar en mi cuenta el importe correspondiente a mi suscripción anual.

FORMA DE PAGO

(1) Transferencia bancaria al recibo de la factura

Cheque nominal al recibo de la factura

(2) Contra tarjeta de crédito

(1) A la cuenta de La Caixa 2100-0424-30-0200041762
(2) Para suscriptores fuera de España

\section{BOLETIN DE DOMICILIACIÓN BANCARIA}

Nombre y apellidos del titular:

Sr. Director del Banco/Caja:

Señor: Le agradeceré que, con cargo a mi cuenta/libreta, atiendan, hasta nueva orden, el recibo que anualmente le presentará la administración de la revista Enseñanza de las Ciencias para el pago de la suscripción.

Banco/Caja:

\begin{tabular}{ll} 
Agencia: & Dirección: \\
\hline & \\
\hline
\end{tabular}

Población: Provincia:

Dígitos de la cuenta:

Fecha:

Nombre y apellidos

Número de tarjeta

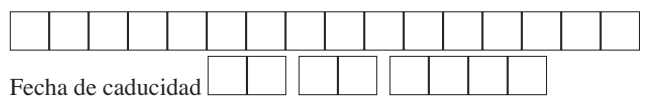

Firma

Dirección, suscripciones y recepción de trabajos:

ICE de la Universitat Autònoma de Barcelona

Edificio A. 08193 Bellaterra (Barcelona)

Teléfono: (3) 58123 98. Fax: (3) 5814656

Web: http://blues.uab.es/rev-ens-ciencias

Administración: r.ensenanza.ciencias@uab.es

Diseño: Jordi Fornas

Maquetación: O3 BCN PACKAGERS S.L.

Impresión: Novoprint, SA

Depósito legal: B. 12.373-1983

Periodicidad: 3 números anuales ISSN: 0212-4521

La revista Enseñanza de las Ciencias está indexada en las bases de datos siguientes: CSIC (ISOC) Consejo Superior de Investigaciones Científicas.

CREDI/OEI Centro de Recursos Documentales e Informáticos/

Organización de Estados Iberoamericanos

y PSYCLIT de American Psychological Association. 


\section{INVESTIGACIÓN DIDÁCTICA}

El pensamiento de orden superior en las clases de ciencias: objetivos, medios y resultados de investigación

Zohar, Anat

La enseñanza de ciencias basada en la elaboración de modelos

Justi, Rosária

La reducción funcional en la justificación de la adiabaticidad de las ondas sonoras

Utges, Graciela y Welti, Reinaldo

Resolver situaciones problemáticas en genética para modificar

las actitudes relacionadas con la ciencia

Martínez Aznar, M. Mercedes e Ibáñez Orcajo, M. Teresa

Las imágenes geológicas y geocientíficas en libros didácticos de ciencias

Silva, Fernanda Keila Marinho da y Compiani, Maurício

Análisis de problemas de selectividad de equilibrio químico: errores

y dificultades correspondientes a libros de texto, alumnos y profesores

Quílez, Juan

La introducción de los conceptos relativos al azar y la probabilidad

en libros de texto universitarios

Barragués Fuentes, José Ignacio y Guisasola Aranzabal, Jenaro

Investigación sobre juegos, interacción y construcción de conocimientos matemáticos

Edo, Mercè y Deulofeu, Jordi

Una propuesta sobre enseñanza de la relatividad en el bachillerato como motivación para el aprendizaje de la física

Pérez, Héctor y Solbes, Jordi

Proceso de simbolización del concepto de potencia: análisis de libros de texto de secundaria Martínez García, Catalina y Penalva Martínez, M. Carmen

\section{INFORMACIÓN BIBLIOGRÁFICA Y NOTICIAS}

Tesis didácticas

Noticias 